Eran Bornstein*, Moti Gulersen, Gregg Husk, Amos Grunebaum, Matthew J. Blitz, Timothy J. Rafael, Burton L. Rochelson, Benjamin Schwartz, Michael Nimaroff and Frank $A$. Chervenak

\title{
Early postpartum discharge during the COVID-19 pandemic
}

https://doi.org/10.1515/jpm-2020-0337

Received July 16, 2020; accepted August 11, 2020; published online August 26, 2020

\section{Abstract}

Objectives: To report our experience with early postpartum discharge to decrease hospital length of stay among low-risk puerperium patients in a large obstetrical service during the COVID-19 pandemic in New York.

Methods: Retrospective analysis of all uncomplicated postpartum women in seven obstetrical units within a large health system between December 8th, 2019 and June 20th, 2020 . Women were stratified into two groups based on date of delivery in relation to the start of the COVID-19 pandemic in New York (Mid-March 2020); those delivering before or during the COVID-19 pandemic. We compared hospital length of stay, defined as time interval from delivery to discharge in hours, between the two groups and correlated it with the number of COVID-19 admissions to our hospitals. Statistical analysis included use of Wilcoxon rank sum test and Chi-squared test with significance defined as p-value $<0.05$.

Results: Of the 11,770 patients included, 5,893 (50.1\%) delivered prior to and 5,877 (49.9\%) delivered during the COVID-19 pandemic. We detected substantial shortening in postpartum hospital length of stay after vaginal delivery (34 vs. $48 \mathrm{~h}, \mathrm{p} \leq 0.0001$ ) and cesarean delivery (51 vs. $74 \mathrm{~h}, \mathrm{p} \leq 0.0001$ ) during the COVID-19 pandemic.

*Corresponding author: Eran Bornstein, MD, Department of Obstetrics and Gynecology, Lenox Hill Hospital - Northwell Health, 100 East 77th St., New York, NY, 10075, USA, E-mail: ebornstein@northwell.edu Moti Gulersen, Timothy J. Rafael, Burton L. Rochelson and Michael Nimaroff, Department of Obstetrics and Gynecology, North Shore University Hospital - Northwell Health, Manhasset, NY, USA Gregg Husk, Amos Grunebaum and Frank A. Chervenak, Department of Obstetrics and Gynecology, Lenox Hill Hospital - Northwell Health, New York, NY, USA

Matthew J. Blitz and Benjamin Schwartz, Department of Obstetrics and Gynecology, Southside Hospital - Northwell Health, Bay Shore, NY, USA
Conclusions: We report successful implementation of early postpartum discharge for low-risk patients resulting in a significantly shorter hospital stay during the COVID-19 pandemic in New York. The impact of this strategy on resource utilization, patient satisfaction and adverse outcomes requires further study.

Keywords: early discharge; length of stay; postpartum; pregnancy; puerperium; SARS-CoV-2.

\section{Introduction}

Shortly after the World Health Organization declared the outbreak of the severe acute respiratory distress syndrome coronavirus 2 (SARS-CoV-2), a global pandemic, New York emerged as the epicenter of the United States (US) [1]. As a result, hospitals within the New York metropolitan area saw a sudden influx of pregnant and nonpregnant patients with complications related to the novel coronavirus disease (COVID-19) [2]. In the face of this a public health emergency, significant changes in patient care were urgently needed in order to optimize care for obstetrical patients, accommodate the high influx of non-obstetrical COVID-19 admissions requiring increased hospital resources, as well as to decrease the potential exposure of both patients and medical staff in the hospital [3-6].

We therefore implemented early discharge of low risk puerperium patients following uncomplicated vaginal and cesarean deliveries, with the intention of reducing potential exposure of patients and medical staff to SARS-CoV-2, facilitate lower patient census to decrease crowding in postpartum units, and accommodate the surging need for beds for nonpregnant patients hospitalized with COVID-19 [4]. Our objective in this study was to demonstrate that implementation of early postpartum discharge was successful in shortening hospital length of stay (LOS) among low-risk obstetrical patients following uncomplicated vaginal or caesarean delivery during the COVID-19 pandemic in New York. 


\section{Materials and methods}

This was a retrospective, cross-sectional, cohort study of all low-risk women who had uncomplicated live births in seven obstetrical units within a large New York based health system including both tertiary care and community hospitals.

Women who had an uncomplicated birth between December 8th, 2019 and June 20th, 2020 were eligible for inclusion. Exclusion criteria included patients with either one or more of the following: operative vaginal deliveries, pregnancy-related complications such as hypertensive disorders of pregnancy, chorioamnionitis, uterine rupture, or hemorrhage requiring transfusion of packed red blood cells, postpartum transfer to another facility or discharge with home care, as well as those with incomplete data regarding the mode of delivery or length of stay. Women were stratified into two groups based on date of delivery in relation to the approximate initial time of the COVID-19 pandemic in New York; i.e., those who delivered before March 15th, 2020 (pre-COVID group) and those who delivered after March 15th, 2020, during the COVID-19 pandemic (COVID group). We compared the hospital LOS, defined as the interval from delivery to discharge time in hours, between the two groups. Additionally, we correlated the length of stay with the number of COVID-19 patients in our hospitals.

Baseline maternal characteristics such as age, body mass index (BMI), race, ethnicity, parity, insurance type and gestational age at delivery were compared between the pre-COVID and COVID groups. Missing data in each categorical variable were excluded from the statistical analysis involving such variable. Statistical analysis included use of Wilcoxon rank sum test and Chi-squared test for comparison of continuous and categorical variables. Statistical significance was defined as $\mathrm{p}$-value $<0.05$.

Of note, early postpartum discharge was implemented at Lenox Hill Hospital on March 12th in anticipation of COVID-19 cases, followed by similar practice in the other hospitals, while an official system-wide policy for implementation was in effect on April 1st. Our guidelines suggested discharge home the day after uncomplicated vaginal deliveries and 2 days after uncomplicated cesarean deliveries using maternal criteria checklists to assure that each patient had no medical conditions or complications requiring longer stay [4]. Due to pediatric considerations and newborn testing requirements, postpartum discharge prior to 1 day was not feasible.

The Northwell Health Institutional Review Board approved this study as minimal-risk research using data collected for routine clinical practice and waived the requirement for informed consent.

\section{Results}

Our obstetrical units delivered 13,644 patients during the study period. Patients with operative vaginal deliveries (256), hypertensive disorders of pregnancy $(1,159)$, chorioamnionitis (190), uterine rupture (9), hemorrhage requiring transfusion (183), transferred to another facility or discharged with home care (153) and missing data (321) were excluded. After applying our exclusion criteria, 11,770 deliveries comprised the study cohort and were further analyzed. Of those, 5,893 (50.1) comprised the pre-
Table 1: Characteristics of the study cohort at baseline.

\begin{tabular}{|c|c|c|c|}
\hline & $\begin{array}{r}\text { Pre-COVID } \\
(n=5893)\end{array}$ & $\begin{array}{r}\text { During-COVID } \\
(n=5877)\end{array}$ & $\begin{array}{l}\mathrm{p}- \\
\text { Value }\end{array}$ \\
\hline $\begin{array}{l}\text { Maternal age, year - } \\
\text { median (IQR) }\end{array}$ & $32(28,35)$ & $32(28,35)$ & 0.39 \\
\hline $\begin{array}{l}\text { BMI, } \mathrm{kg} / \mathrm{m}^{2} \text { - median } \\
\quad(\mathrm{IQR})\end{array}$ & $\begin{array}{r}29.9 \\
(26.7,33.7)\end{array}$ & $\begin{array}{r}29.8 \\
(26.6,33.6)\end{array}$ & 0.16 \\
\hline \multicolumn{4}{|l|}{ Race - n, \% } \\
\hline White & $2,669(45.3)$ & $2,862(48.7)$ & $0.04^{\mathrm{a}}$ \\
\hline Black & $662(11.2)$ & $660(11.2)$ & \\
\hline Asian & 784 (13.3) & $778(13.2)$ & \\
\hline Other/Multiracial & $1,425(24.2)$ & $1,342(22.8)$ & \\
\hline Unknown ${ }^{a}$ & $353(6)$ & $235(4)$ & \\
\hline \multicolumn{4}{|l|}{ Ethnicity - n, \% } \\
\hline Hispanic & $1,127(19.1)$ & $1,059(18)$ & $0.15^{\mathrm{a}}$ \\
\hline Non-Hispanic & $4,700(79.8)$ & $4,735(80.6)$ & \\
\hline Unknown ${ }^{\mathrm{a}}$ & $66(1.1)$ & $83(1.4)$ & \\
\hline \multicolumn{4}{|l|}{ Insurance type - n, \% } \\
\hline Medicaid & $2,148(36.5)$ & $1,968(33.5)$ & 0.001 \\
\hline Other/Unknown & $3,745(63.5)$ & $3,909(66.5)$ & \\
\hline \multicolumn{4}{|l|}{ Parity - n, \% } \\
\hline 0 & $2,502(42.5)$ & $2,494(42.4)$ & 0.31 \\
\hline 1 & $2,059(34.9)$ & $2,026(34.5)$ & \\
\hline$\geq 2$ & $1,328(22.5)$ & $1,355(23.1)$ & \\
\hline \multicolumn{4}{|l|}{ Mode of delivery - $n, \%$} \\
\hline Vaginal delivery & $4,025(68.3)$ & $4,036(68.7)$ & 0.66 \\
\hline Cesarean delivery & $1,868(31.7)$ & $1,841(31.3)$ & \\
\hline $\begin{array}{c}\text { Gestational age at delivery, } \\
\text { weeks - median (IQR) }\end{array}$ & $39(38.3,40)$ & $39(38.4,40)$ & 0.30 \\
\hline
\end{tabular}

BMI, body mass index; IQR, interquartile range. ${ }^{\text {a Unknown data were }}$ not included in statistical analysis.

COVID group, and 5,877 (49.9) the COVID group. Maternal baseline demographics such as age, body mass index, ethnicity, parity, mode of delivery and gestational age at delivery were similar between the two groups (Table 1). Racial distribution was significantly different with slightly more white and less other/multiracial women during COVID compared to pre-COVID (48.7 vs. $45.3 \%$, and 22.8 vs. $24.2 \%$, respectively $\mathrm{p}=0.04$ ) (Table 1 ). There was also a lower proportion of women with Medicaid insurance during COVID compared to pre-COVID (33.5 vs. $36.5 \%$, $\mathrm{p}=0.001$ ) (Table 1). While differences in race and insurance type reached statistical significance, these differences were small, suggesting little, if any, clinical significance (Table 1). The distribution of postpartum median length of stay among uncomplicated vaginal and caesarean deliveries during the study period is presented in Figure 1. A marked decrease in the length of stay is noted from March 2020 onwards. Comparison of the primary outcome between the two groups indicates a statistically significant difference in the median length of stay following vaginal delivery, shortened by $29 \%$ from average of $48 \mathrm{~h}$ (Inter Quartile Range (IQR) 43 and 55) Pre-COVID to 34 h (IQR 28 


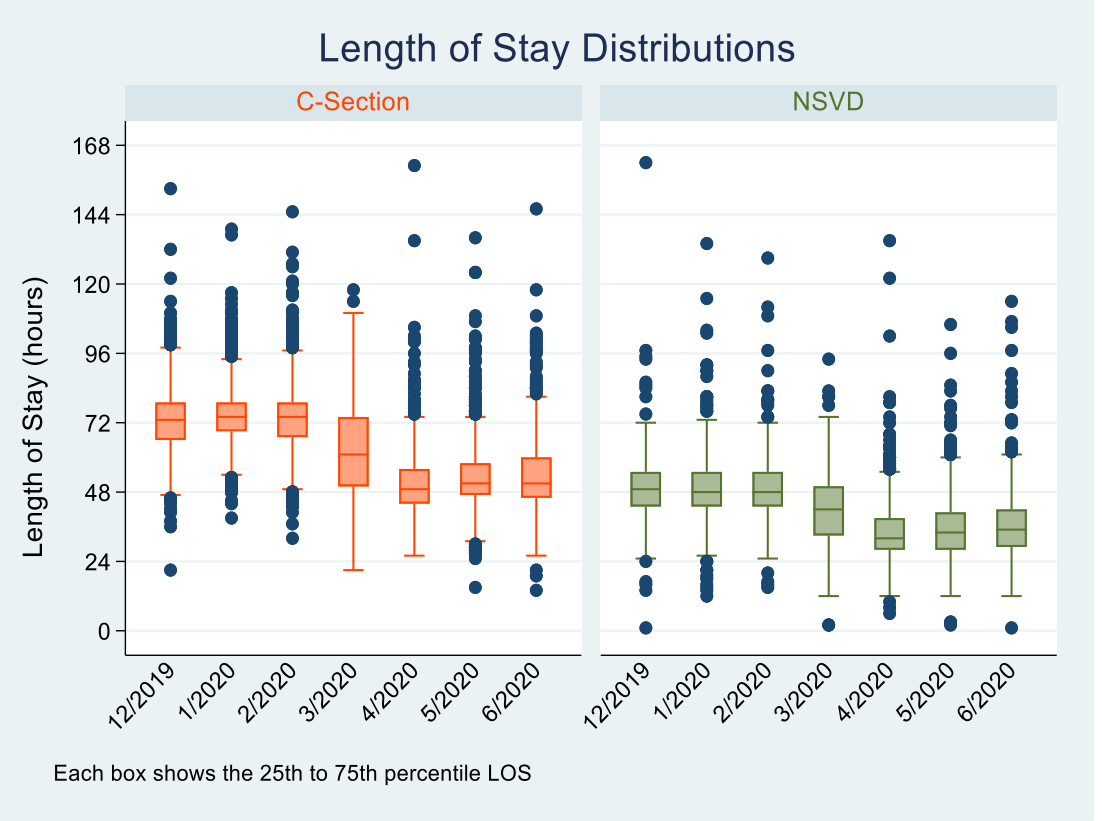

Figure 1: Distributions of length of stay. The boxes show the 25th, 50th and 75th percentile LOS for patients after uncomplicated vaginal and cesarean delivery each month during the study period.
Table 2: Comparison of postpartum length of stay after vaginal and cesarean delivery between the two groups.

\begin{tabular}{lcrl}
\hline & $\begin{array}{r}\text { Pre-COVID } \\
(\mathbf{n = 5 8 9 3 )}\end{array}$ & $\begin{array}{r}\text { During COVID } \\
(\mathbf{n = 5 8 7 7 )}\end{array}$ & p-Value \\
\hline $\begin{array}{l}\text { Vaginal delivery, } \\
\text { hours - median (IQR) }\end{array}$ & $48(43,55)$ & $34(28,41)$ & $<0.0001$ \\
$\begin{array}{l}\text { Cesarean delivery, } \\
\text { hours - median (IQR) }\end{array}$ & $74(67,79)$ & $51(46,58)$ & $<0.0001$ \\
\hline
\end{tabular}

and 41) during COVID ( $\mathrm{p} \leq 0.0001)$. Similarly, length of stay following caesarean delivery shortened by $31 \%$, from an average of $74 \mathrm{~h}$ (IQR of 67 and 79) pre-COVID to $51 \mathrm{~h}$ (IQR of 46 and 58) during COVID ( $\leq \leq 0.0001)$ (Table 2). Data regarding the median length of stay of uncomplicated deliveries (caesarean and vaginal) throughout the study period in the context of the total daily inpatient census of COVID-19 patients at the seven study hospitals is presented in Figures 2 and 3, respectively. The decrease in

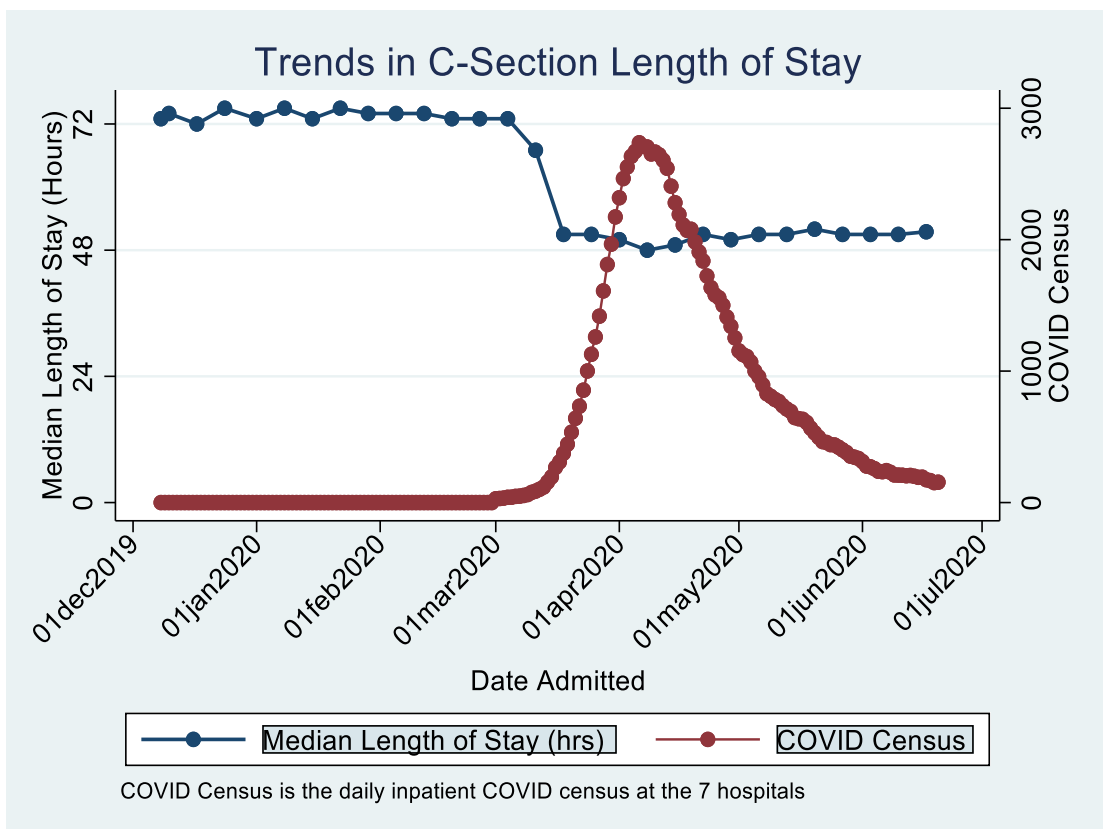

Figure 2: Median length of stay for patients after uncomplicated cesarean delivery. This graph provides the pandemic context by showing the total daily inpatient census of COVID-19 patients at the seven study hospitals. 


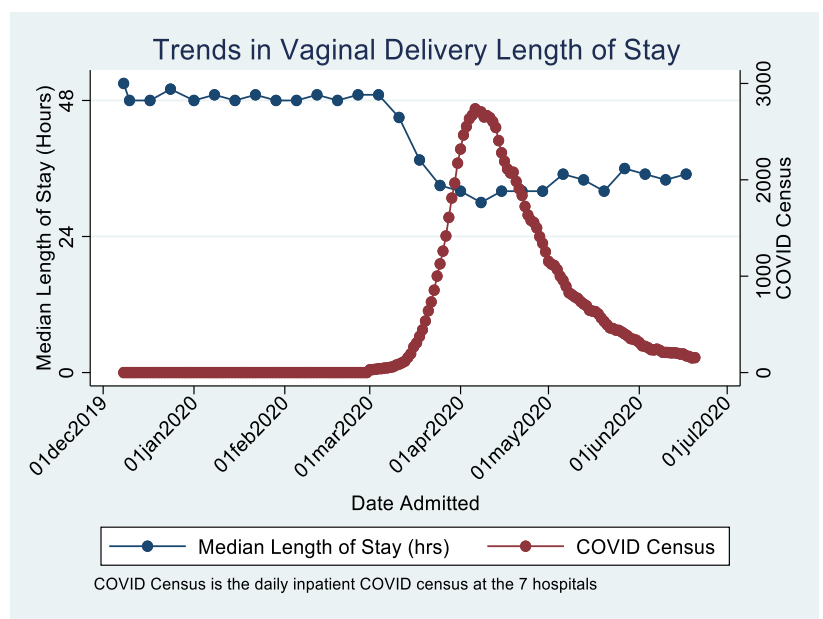

Figure 3: Median length of stay for patients after uncomplicated vaginal delivery.

This graph provides the pandemic context by showing the total daily inpatient census of COVID-19 patients at the seven study hospitals.

postpartum length of stay after uncomplicated vaginal and cesarean delivery coincided with the abrupt increase in inpatient census of COVID-19 patients within our hospitals.

\section{Discussion}

Our study demonstrates a significant shortening in the hospital length of stay during the COVID-19 pandemic for low-risk puerperium patients following uncomplicated vaginal or cesarean delivery in a large health system. Specifically, during the COVID-19 pandemic, the median postpartum LOS decreased by approximately $30 \%$, with LOS shortened by average of $14 \mathrm{~h}$ after vaginal delivery and by $23 \mathrm{~h}$ following cesarean delivery compared to preCOVID. This strategy had several potential implications that benefited the patients, obstetrical service, and hospital. From an infection control perspective, decreasing the length of stay for the patient, and thus her and baby, reduced the potential exposure to SARS-CoV-2 for patients and medical staff, as well as decreased overall crowding in the postpartum units by allowing for more patients to utilize single rooms when available. In addition, it increased our ability to allocate resources such as hospital beds and medical personnel from obstetrical units to non-obstetrical services to accommodate for increasing hospital demands by the surge of COVID-19 admissions.

Shortening postpartum length of stay in hospitals as a strategy for cost containment and hospital bed availability was conceptualized in the United States decades ago [7-
10]. Since then, however, robust clinical evidence and full economic evaluations are lacking [11-16]. A recent systematic review investigating the impact of early postpartum discharge after vaginal delivery demonstrated the lack of support or evidence against the widespread use of early discharge [11]. The authors reported no statistical difference in maternal and neonatal morbidity and maternal and neonatal readmission rates [11]. In the setting of an infectious disease pandemic, and lack of high quality evidence demonstrating risks associated with early postpartum discharge, our strategy should be considered as a reasonable option to reduce exposure and reallocating hospital resources.

There are several strengths to this study. First, to our knowledge, this is the first study investigating the feasibility of a system-wide initiative to shorten puerperium in-hospital length of stay by expediting early discharge for uncomplicated deliveries during the COVID-19 pandemic. Secondly, we present robust data that is based on a population from the largest health system in the northeast US, and includes over 30,000 annual deliveries from both tertiary care and community hospitals. Moreover, this population is diverse in terms of maternal demographic, racial, and ethnic characteristics and is thus, generalizable.

Our study has a few limitations. There was heterogeneity in documentation of hemorrhage and blood transfusion between the different hospitals, thus this outcome was not available for all patients. However, it was included for patients from three large sites, which represented the majority (68\%) of deliveries. In addition, given the retrospective design with information based on review of medical records, not all charts had complete data available regarding pregnancy-related complications. Thus, it is likely that our population is not entirely low-risk and includes some higher risk patients or complicated deliveries.

In conclusion, our data demonstrate that an early postpartum discharge initiative was successful in significantly reducing hospital length of stay for puerperium women with uncomplicated vaginal and cesarean deliveries. Further review of this strategy in terms of patient safety, potential adverse outcomes, utilization of healthcare resources, and patient satisfaction, should be performed to further solidify the benefits of early postpartum discharge. Nevertheless, during the unprecedented time of the COVID-19 pandemic, this enabled our institution to maintain the obstetrical service active, while minimizing patient and medical personnel exposure to SARS-CoV-2, and allowed for better allocation of healthcare resources. 
Acknowledgments: We would like to acknowledge the contributions of the Northwell Health COVID-19 Research Consortium.

Research funding: None declared.

Author contributions: All authors have accepted responsibility for the entire content of this manuscript and approved its submission.

Competing interests: Authors state no conflict of interest. Ethical approval: The Northwell Health Institutional Review Board approved this study as minimal-risk research using data collected for routine clinical practice and waived the requirement for informed consent.

\section{References}

1. McKinley J New York city region is now an epicenter of the coronavirus pandemic. New York: The New York Times; 2020. Available from: https://www.nytimes.com/2020/03/22/ nyregion/Coronavirus-new-York-epicenter.html [Accessed 22 Mar 2020].

2. Tekbali A, Grunebaum A, Saraya A, McCullough L, Bornstein E, Chervenak FA. Pregnant vs. nonpregnant severe acute respiratory syndrome coronavirus 2 and coronavirus disease 2019 hospital admissions: the first 4 weeks in New York. Am J Obstet Gynecol 2020 Jul. [Epub ahead of print].

3. Chervenak FA, Grunebaum A, Bornstein E, Wasden S, Katz A, Rochelson BL, et al. Expanding the concept of professional integrity of obstetrics during a public health emergency. J Perinat Med 2020;48:435-7.

4. Rochelson B, Nimaroff M, Combs A, Schwartz B, Meirowitz N, Vohra $\mathrm{N}$, et al. The care of pregnant women during the COVID-19 pandemic - response of a large health system in metropolitan New York. J Perinat Med 2020;48:453-61.
5. Narang K, Ibirogba ER, Elrefaei A, Trad ATA, Theiler R, Nomura R, et al. SARS-CoV-2 in pregnancy: a comprehensive summary of current guidelines. J Clin Med 2020;9:1521.

6. Dashraath P, Wong JLJ, Lim MXK, Lim LM, Li S, Biswas A, et al. Coronavirus disease 2019 (COVID-19) pandemic and pregnancy. Am J Obstet Gynecol 2020;222:521-31.

7. Fink AM. Early hospital discharge in maternal and newborn care. J Obstet Gynecol Neonatal Nurs 2011;40:149-56.

8. Brooten D, Roncoli M, Finkler S, Arnold L, Cohen A, Mennuti M. A randomized trial of early hospital discharge and home follow-up of women having cesarean birth. Obstet Gynecol 1994;84: 832-8.

9. Strong TH, Brown WL, Brown WL, Curry CM. Experience with early postcesarean hospital dismissal. Am J Obstet Gynecol 1993;169: $116-9$.

10. Brooten D, Kumar S, Brown LP, Butts P, Finkler SA, BakewellSachs $S$, et al. A randomized clinical trial of early hospital discharge and home follow-up of very-low-birth-weight infants. N Engl J Med 1986;315:934-9.

11. Benahmed N, San Miguel L, Devos C, Fairon N, Christiaens W. Vaginal delivery: how does early hospital discharge affect mother and child outcomes? A systematic literature review. BMC Pregnancy Childbirth 2017;17:289.

12. Brown S, Small R, Faber B, Krastev A, Davis P. Early postnatal discharge from hospital for healthy mothers and infants. Cochrane Database Syst Rev 2002:CD002958.

13. Sainz Bueno JA, Romano MR, Teruel RG, Benjumea AG, Palacin AF, Gonzalez CA, et al. Early discharge from obstetrics-pediatrics at the hospital de Valme, with domiciliary follow-up. Am J Obstet Gynecol 2005;193:714-26.

14. Kehila M, Magdoud K, Touhami O, Abouda HS, Jeridi S, Marzouk SB. Early postpartum discharge: outcomes and risk factor readmission. Pan Afr Med J 2016;24:189.

15. Brumfield CG. Early postpartum discharge. Clin Obstet Gynecol 1998;41:611-25.

16. Grullon KE, Grimes DA. The safety of early postpartum discharge: a review and critique. Obset Gynecol 1997;90:860-5. 